\title{
1) Vorbemerkungen
}

Ziel des folgenden Beitrags ist die Analyse von politischen und ökonomischen Grenzen und Möglichkeiten der wirtschaftspolitischen Alternative, die im französischen Gemeinsamen Regierungsprogramm (GRP) enthalten war. Das GRP war die am weitesten entwickelte wirtschaftspolitische Alternative, die von linken politischen Parteien in Westeuropa bisher vorgelegt wurde. Gedacht war das GRP als eine wichtige Etappe auf einem nationalspezifischen Weg zu einer französischen Form des Sozialismus. Im politischen Bereich hatte es die Aufgabe, die politischen Positionen der drei Parteien zu vereinheitlichen, die das GRP unterzeichnet haben. Diese Aufgabe hat es im Wahlkampf nicht überstanden. Die „Dynamik der Einheit", die vom GRP zunächst freigesetzt wurde, ist von den Profilierungsstrategien der Parteiapparate zerstört worden.

Um zu einem Verständnis des Bruchs in der Linksunion zu kommen, werden zunächst dessen Ursachen im französischen Wahlsystem und den Wahlkampfstrategien der Parteiapparate ausgelotet. Im folgenden dritten Teil wird versucht aufzuzeigen, aus welchen wirtschaftspolitischen Problemen und sozialpolitischen Defiziten der jüngeren französischen Wirtschaftsentwicklung die Reformforderungen des GRP entstanden sind. Im vierten Teil werden dann die im engeren Sinne ökonomischen Grenzen und Möglichkeiten des GRP untersucht.

Der Beitrag konzentriert sich im wesentlichen auf die politische und ökonomische Alternative des GRP. Die komplexen gewerkschaftlichen Strategien gegenïber dem GRP werden nur am Rande erwähnt. Die weitergehende gesellschaftspolitische Alternative der ,autogestion“, d.h. der französischen Varianten einer rätedemokratischen Arbeiterselbstverwaitung, die insbesondere von der Gewerkschaft CFDT aufgegriffen und weiterentwickelt, aber auch von den politschen Parteien diskutiert wurde, kann in diesem Beitrag ebenfalls nicht behandelt werden.

\section{2) Der Bruch der Linksunion und die selbstverschuldete Wahiniederlage}

Am 22. September 1977 ist die französische Linksunion an der Aktualisierung ihres GRP gescheitert. Die Sozialistische Partei (SP), die Kommunistische Partei (KP) und die Splitterpartei-Bewegung der radikalen Linken konnten sich auf die Fortschreibung ihres Programms vor allem in einem Punkt nicht einigen: Die Zahl der Tochter- 
gesellschaften von Industriegruppen, die verstaatlicht werden sollten. Weitere Punkte, die die atomare Bewaffnung Frankreichs, die unterschiedlichen Auffassungen über die Finanzierung des GRP, Einkommensverteilung, Institutions- und Wahlrechtsprobleme und vieles andere mehr haben in der Kontroverse zwischen SP und KP vom September 1977 bis März 1978 nur eine untergeordnete Rolle gespielt.

Das GRP war ein Programm, dessen politische Funktion auf eine vereinheitlichte Wahlkampfstrategie der drei Vertragsparteien gerichtet war. Eine „Dynamik der Einheit " sollte geschaffen werden, die den Wahlsieg ermöglichen sollte. Das GRP, das 1972 unterzeichnet wurde, hat diese Aufgabe erfült. 1974 ist Mitterrand, der Generaisekretär der SP, bei den Präsidentschaftswahlen nur knapp unterlegen. Bei Kommunalwahlen im Frühjahr 1977 konnte die Linksunion eine große Zahl von wichtigen Städten erobern. Im September 1977 - vor dem Bruch der Linksunion konnte sie 1t. Meinungsumfragen rd. $56 \%$ der Wählerintentionen auf sich vereinigen. Die äußerst scharf geführte Polemik zwischen SP und KP hat die „Dynamik der Einheit ${ }^{66}$ zerstört. Im ersten Wahlgang hat die Linke nur rd. $49 \%$ der Wählerstimmen auf sich vereinigen können.

Das GRP war über die reformorientierten Inhalte hinaus in erster Linie ein Wahlprogramm. Den Linksparteien ist ebenso wie den Rechtsparteien die Koppelung ihrer Wahltaktik an präzise gesellschaftspolitische Inhalte zumindest nach den verlorenen Wahlen eher hinderlich als vorteilhaft. Eine Neuauflage oder Aktualisierung des GRP ist nicht mehr beabsichtigt. Das liegt in den Besonderheiten der politischen Sphäre integrierter bürgerlich-kapitalistischer Systeme begründet. Unter Durchschnitîsbedingungen wirtschaftiiicher Stabilität, die von der Höhe der Arbeitslosenquote wesentlich geprägt wird, ist das Wählerverhalten auch langfristig erstaunlich beständig. Stimmengewinne sind nur schwer zu erzielen. Sie werden in beschränktem Umfang möglich, wenn programmatische Vorstellungen von den jeweils konkurrierenden Parteien übernommen werden. Ein ausformuliertes Programm wie das GRP hat in dieser Konstellation vor allem zwei Nachteile. Es ist ein Kompromiß für die Parteien der Linksunion gewesen, mit denen weite Teile der Parteibasis und der Stammwähler nur bedingt einverstanden waren, insbesondere in der KP. Damit ist es zum Auslöser von Konflikten innerhalb der Parteien der Linksunion geworden. Die festgeschriebene Programmatik war bei der Gewinnung neuer Wählerschichten nur bedingt, d.h. für die SP, nicht aber für die KP von Vorteil. Daran ist die Linksunion letztendlich gescheitert.

Die Linksunion hat sich nach den verlorenen Wahlen in ihrem Kern als Wahlbündnis zweier Parteiapparate zu erkennen gegeben. Geschickt wurde das Bedürfnis nach Einheit der Linken in den Parteien und Gewerkschaften, das im Bewußtsein weiter Teile der französischen Wähler Voraussetzung der Regierungsübernahme durch die Linke isî, in der ersten Phase nach 1972 genutzt. Die französische Wählerschaft zerfällt in drei Blöcke : ein Drittel links, ein Drittel rechts und ein Drittel, der sogenannte ,Sumpf“ der Wechselwähler. Diese Teilung geht bis weit ins 19. Jahrhundert zurïck. Trotz tiefgreifender Strukturänderungen in Wirtschaft und Gesellschaft hat sich an diesem Wählerverhalten in Zeiten relativ ungestörter wirtschaftlicher Entwicklung nicht viel geändert. Die französische Rechte übt seit 1958, als 
de Gaulle an die Macht kam, ohne Unterbrechung nahezu uneingeschränkt die Regierungsgewalt aus. De Gaulle hatte 1958 die parlamentarische Demokratie mit Verhältniswahlrecht in eine Präsidentialdemokratie mit Mehrheitswahlrecht verwandelt. Die gaullistische Partei konnte sich mit populistischen Thesen durchsetzen und aus dem „Sumpf“, dem Drittel der Wechselwähler, die zur konservativen Mehrheitsbildung erforderlichen Stimmen binden.

Das Mehrheitswahlsystem hat bei der Festigung konservativer Mehrheiten im französischen Parlament und damit für die Bildung einer konservativen Regierung eine wesentliche Funktion. Wahlkreis-Arithmetik, d.h. die Festlegung der Wahlkreise, erlaubt es, konservative Abgeordnete in vielen Fällen mit einer erheblich geringeren Stimmenzahl ins Parlament zu schicken. Gewählt wird in zwei Wahlgängen. Im ersten Wahlgang wird der Kandidat gewählt, der die absolute Mehrheit der abgegebenen Stimmen auf sich vereinigt. Nur rd. 1/7 der Kandidaten sind in der vergangenen Wahl im ersten Wahlgang durchgekommen. Im zweiten Wahlgang ist gewählt, wer die einfache Mehrheit der abgegebenen Stimmen erzielt. Für die Linke wie für die Rechte bedeutet dies, daß sich der oder die jeweils schlechter plazierten Kandidaten zurückziehen und nur der jeweils erstplazierte Kandidat im Rennen bleibt. Das Mehrheitswahlsystem hat zweifelsohne dazu beigetragen, Frankreich in zwei Blöcke, einen linken und einen rechten, zu spalten. Es zwingt aber auch den Parteien innerhalb eines Blocks zwei einander widersprechende Strategien auf. Vor dem ersten Wahlgang müssen sich die Parteien profilieren, gegebenenfalls auch zu Lasten ihrer linken Konkurrenten, um ihre Kandidaten innerhalb des linken Blocks an die erste Stelle zu bringen. Eine Woche später, im zweiten Wahlgang, müssen sie sich einigen, damit die Stammwähler des Zweitplazierten ihre Stimmen auf den Erstplazierten übertragen. Gut funktionierende Stimmenübertragungen innerhalb des linken und des rechten Blocks sind wahlentscheidend: 57 von 491 Abgeordneten sind im zweiten Wahlgang mit einer Mehrheit von unter $1 \%$ vor dem Kandidaten des gegnerischen Blocks gewählt worden.

Die offiziellen Wahlergebnisse des ersten Wahlgangs sind aus der Tabelle $1 \mathrm{er}$ sichtlich.

Die Linke hat mit rd. $49 \%$ gegenüber der Rechten zwar einen klaren Sieg davongetragen, sie hat jedoch die zum Sieg im zweiten Wahlgang erforderlichen $52.53 \%$ nicht erreicht. Mit rd. $46 \%$ liegt die Rechte gute 2 Prozentpunkte zurück. Die von ihr befürchtete Legitimitätsfalle jedoch hat sich nicht geöffnet. Ein ernstes Legitimitätsproblem für das politische System Frankreichs wäre entstânden, wenn die Linke im ersten Wahlgang knapp über $50 \%$ der Wählerstimmen auf sich vereinigt, d.h. die Mehrheit der französischen Bevölkerung hinter sich hätte, die Rechte aber auf Grund der Wahlkreis-Arithmetik im zweiten Wahlgang im Parlament eine knappe Mehrheit der Abgeordneten durchgebracht hätte. Soziale Unruhen sind für diesen Fall nicht ausgeschlossen worden.

Tabelle 2 zeigt nun, daß die Rechte diesem Schicksal entgangen ist.

Das französische Wahlsystem hat die Niederlage der Rechten im ersten Wahlgang in einen komfortablen Wahlsieg im zweiten Wahlgang verwandelt. Der Rechten wurde 
Tabelle 1: Anteile der Parteien im 1. Wahlgang

Extreme Linke

KP

$S P$

BRL
3.33

20.55

22.58

2.11
48.57
Gaullisten

Giscardisten
22.62

23.84
46.46
Ökologen

Sonstige*
2.14

2.77

* Die ,Sonstigen" können zu je rd. 1 \% der Linken und der Rechten, der Rest der extremen Rechten zugeordnet werden.

Quelle: Le Monde, La defaite de la gauche, 1978, S. 76

Tabelle 2: Verteilung der Parlamentssitze im 2. Wahlgang

\begin{tabular}{|c|c|c|c|c|c|c|}
\hline Parteien & $\begin{array}{l}\text { Altes Parla- } \\
\text { ment }\end{array}$ & $\begin{array}{r}\text { neu g } \\
\text { 1. Wahlgg. }\end{array}$ & $\begin{array}{l}\text { wählt } \\
\text { 2. Wahlgg. }\end{array}$ & $\begin{array}{l}\text { Neues Parla- } \\
\text { ment }\end{array}$ & $\begin{array}{l}\text { Linke/Rechte } \\
\text { insgesamt }\end{array}$ & $\begin{array}{l}\text { Zugänge } \\
\text { Abgänge }\end{array}$ \\
\hline $\mathrm{KP}$ & 74 & 4 & 82 & 86 & & +12 \\
\hline SP & 95 & 1 & 103 & 104 & & +9 \\
\hline$B R L$ & 13 & - & 10 & 10 & & -3 \\
\hline Sonstige & 2 & - & 1 & 1 & (201) & -1 \\
\hline Gaullisten. & 173 & 31 & 119 & 150 & & -23 \\
\hline Giscardisten & 119 & 32 & 106 & 138 & & +17 \\
\hline Sonstige & 15 & - & 2 & 2 & (291) & -13 \\
\hline Insgesamt & 491 & 68 & 423 & 491 & 491 & - \\
\hline
\end{tabular}

Quelle: Le Monde, La defaite de la gauche, S. 89

ein Vorsprung von 90 Abgeordneten beschert. Der Reformpolitik des Präsidenten Giscard d'Estaing, von der kennzeichnenderweise seit seiner Wahl 1974 nichts verwirklicht wurde, würden im politischen System auch in Zukunft keine Hindernisse mehr entgegenstehen (1).

Das französische Wahlsystem enthält einen Profilierungszwang und einen Einigungszwang. Es hat die Konflikte zwischen SP und BRL auf der einen und der KP auf der anderen Seite gewiß nicht hervorgebracht, aber es hat bestehende Divergen-

1 In einer kleinen Schrift, die uraltes konservatives Gedankengut Frankreichs nicht ungeschickt modernisiert, entwirft der französische Präsident ein neoliberales Konzept mit Reformen wie Dezentralisierung, neues qualitatives Wachstum etc. Bisher wurde von diesem Konzept nicht einmal ein Ansatz verwirklicht. Giscard d'Estaing, V., Democratie Francaise, Paris 1976 
zen verschärft. Profilierungstendenzen und .Einigungsbestrebungen kennzeichnen die französische Arbeiterbewegung seit der Gründung der KP auf dem Kongreß von Tours (2). Die Spaltung der Arbeiterbewegung in einen kommunistischen und einen sozialdemokratischen bzw. sozialistischen Flügel, die nicht nur in Frankreich nach dem ersten Weltkrieg sich durchgesetzt hat, hat die Möglichkeiten gesellschaftlicher Veränderung bis heute in den kapitalistischen Ländern stark eingeschränkt. Der Aufbau von bürokratisch organisierten Parteiapparaten mit fest angestellten, besoldeten Parteiarbeitern hat die Spaltung auch in Frankreich verfestigt.

Dem Kampf um gesellschaftliche Veränderungen werden Grenzen gezogen, die sich aus den Reproduktionserfordernissen des Parteiapparates bestimmen lassen: Reproduktionserfordernisse ideologischer und materieller Natur, die in der feindlichen Umwelt bürgerlich-kapitalistischer Gesellschaften nur schwer zu erfüllen sind. Es kann deshalb nicht davon ausgegangen werden, daß eine Verschärfung der Klassenauseinandersetzungen die ideologische und materielle Reproduktion des Parteiapparates sicherstellt, sondern sie vielmehr gefährdet. Professionalisierte Parteiapparate entwickeln vorzugsweise politische Strategien, die ihr Reproduktionsinteresse nicht in Frage stellen. Tendenziell favorisieren sie deshalb politische Strategien, die den Bestand bürgerlich-kapitalistischer Produktionsverhältnisse, aus denen sie ihre Reproduktionserfordernisse ziehen, günstigstenfalls vordergründig in Frage stellen. Der Parteiapparat der KP reproduziert sich materiell über Mitgliederbeiträge, Spenden, Verkäufe von Publikationen bzw. wesentlich durch Einkünfte aus Geschäftsunternehmen, insbesondere Export-Import-Unternehmen, die mit sozialistischen Ländern Handel treiben. Der Parteiapparat reproduziert sich, indem er seine ökonomische Basis, seinen Mitgliederbestand und sein Wählerpotential mindestens auf „einfacher Stufenleiter" sicherstellt. Er reproduziert sich ideologisch durch die Weiter" entwicklung und Verbreitung einer in seinem Selbstverständnis , revolutionären“ Theorie, die in unterschiedlichen Graden von Vertiefung auch an Parteischulen vermittelt wird. Sie besteht heute für die französische KP aus einer nationalspezifischen Ausprägung der „Theorie des staatsmonopolistischen Kapitalismus ${ }^{66}$, die mindestens den Vorteil hat, daß sie nicht mit den Reproduktionsbedürnissen des Parteiapparates in Konflikt gerät. Die Klassenanalyse liest sich dann auch folgerichtig auf weiten Strecken wie eine Arbeitsmarktstudie, in der nach „Schichten“ Ausschau gehalten wird, in denen neue Mitglieder geworben oder die in ein „breites antimonopolitisches Bündnis“" aufgenommen werden können (3). Ein besonderes Problem der französischen KP ist die schnelle Rotation insbesondere der jüngeren Mitglieder, die nur wenige Jahre in der Partei bleiben. Elleinstein, ein kommunistischer Parteihistoriker, setzt sich mit diesem Problem anhand parteioffizieller Quellen auseinander und kommt zu einem jährlichen Mitgliederschwund von rd. $10 \%$, der aber zu niedrig sein dürfte (4).

2 Auf dem Gründungskongreß der KP hat die Unterstützung der Oktoberrevolution bzw. Sowjetunion eine wichtige, wenn nicht die ausschlaggebende Rolle gespielt. Siehe Freville, Jean, La nuit finit a Tours, Paris 1970, S. $177 \mathrm{ff}$.

3 Boccara, Paul, u.a., Der staatsmonopolitische Kapitalismus, Frankfurt 1972. Hier das Kapitel III, Die Klassen der Gesellschaft, S. $154 \mathrm{ff}$.

4

Elleinstein, Jean, Le PC, Paris 1976, S. $98 / 99$ 
Sicher ist, daß in Frankreich helite die Zahl der ehemaligen Mitglieder ein mehrfaches der gegenwärtig eingeschriebenen Mitglieder beträgt. Im Juni 1976 hatte die KP rd. 500000 Mitglieder. Über gegenwärtige Mitgliederzahlen liegen keine verläßlichen Informationen vor. Die ehemaligen Mitglieder der KP spielen zweifelsohne bei der Bewußstseinsbildung der französischen Bevölkerung eine wichtige Rolle. Nicht zuletzt über die ehemaligen Mitglieder hat die KP das Bewußtsein der französischen Bevölkerung von den Möglichkeiten gesellschaftlicher Veränderung, das in der Bundesrepublik nach dem Zweiten Weltkrieg nicht wieder entwickelt werden konnte, wachgehalten - wenn auch in diesem Fall auf einem Wege, der vom selbst. gesteckten Ziel der Partei weit wegführt.

Die Sozialistische Partei ist eine Gruindung aus unterschiedlichen sozialdemokratischen oder sozialistischen Strömungen, die sich 1971 zusammengefunden haben. François Mitterrand, der sich als ,charismatischer "Staatsmann gebärdet und durch die Medien als soicher feiern läßt, ist ihr erster Sekretär, der über diese Partei politische Ambitionen durchsetzen will. Er hat wesentlich dazu beigetragen, die Partei auf ejn Wahlergebnis knapp hinter den Gaullisten zu führen (Tabelle 1). Innerhalb der Partei wird er vom linken Flügel, dem CERES, politisch belkämpft, der sich die nichtgelöste Aufgabe gestellt hatte, die Verbindung zur K.P politisch zu gestalten (5). Die Reproduktionsinteressen der SP gehen über die der KP hinaus. Die SP produziert zusätzlich das Image des Staatsmannes Mitterrand, der als Präsidentschaftskandidat aufgebaut wird. Die SP sucht neue Wählerreservoirs nach rechts, aber auch unter den traditionell kommunistischen Wählern. Sie gerät dabei notwendig mit der $\mathrm{KP}$ in Konflikt, die bei den Präsidentschaftswahlen 1981 diesmal einen eigenen Kandidaten, höchstwahrscheinlich Marchais, aufstellen wird. In den knapp verlorenen Präsidentschaftswahlen 1974 war Mitterrand dagegen der einzige Kandidat der Linksunion.

Die Spaltung der Arbeiterbewegung nach dem Ersten Weltkrieg bzw. der Oktoberrevolution ist in Frankreich nicht Ausdruck organisationspolitischen Fehlverhaltens gewesen. Sie ist aus den wirtschaftlichen und politischen Entwicklungsprozessen des letzten Drittels des 19. Jahrhunderts freigesetzt worden. Die nationale und internationale Ausbreitung der kapitalistischen Produktionsweise ging mit der Verallgemeinerung des Lohnarbeitsverhältnisses einher, die gleichzeitig verbunden war mit der Abschottung nationaler Arbeitsmärkte und deren Segmentierung in Arbeiter, Angestellte, Branchen, Gewerkschaften etc. Der vereinheitlichenden Tendenz, die durch die Ausbreitung des Lohnarbeitsverhältnisses hervorgerufen wurde, wirkte eine Partikularisierung entgegen, die sich über spezifische Arbeitsmärkte konstituiert und sich jeweils in besonderen Bewußtseinsformen niederschlägt. Die Organisationen der Arbeiterbewegung haben auch in Frankreich auf der politischen und der gewerkschaftlichen Ebene aus den Reproduktionsinteressen ihrer miteinander konkurrierenden Apparate heraus die partikularisierenden und weniger die vereinheitlichenden Tendenzen der wirtschaftlichen und politischen Entwicklung aufgenommen und verfestigt.

5 Eine erste, wenn auch noch nicht sehr grüđliche Geschichte der SP gibt Pfister, Thierry, Les socialistes, Paris 1977. Zur Funktion des CERES siehe S. $44 \mathrm{ff}$. 
Am 22. September 1977 ist die Linksunion an der Aktualisierung ihres GRP gescheitert. Bis zum ersten Wahlgang haben sich die Linksparteien bis aufs Messer bekämpft und sich dem partikula risierenden Profilierungszwang unterworfen. In vorgeblich politischen Auseinandersetzungen sind die wesentlichen Probleme, mit denen die sich verschärfende Weltwirtschaftskrise die französische Bevölkerung in zunehmendem Umfang konfrontiert, allmählich nur noch am Rande erwähnt worden. Die Linke aber kann in Frankreich nur an die Regierung kommen, wenn sie die gleichermaßen in der gesellschaftlichen Entwicklung angelegte Tendenz zur Vereinheitlichung aufgreift und verwirklicht. Die französischen Wähler haben in der Präsidentschaftswahl 1974, in den Kommunalwahlen 1977 und zahlreichen Meinungsumfragen gezeigt, daß sie der ,Dynamik der Einheit" vertrauen. Es ist hier nicht der Ort, die Argumente zu überprüfen, mit denen KP und SP versucht haben, ihren Konfron. tationskurs zu begründen, d.h., ob Mitterrand nach den Kommunalwahlen plötzlich eine Rechtswendung vollzogen oder die KP Order aus Moskau erhalten hat, durch einen möglichen Wahlsieg der Linksunion Westeuropa nicht zu destabilisieren. Die KP hat offerbar von der "Dynamik der Einheit" in weitaus geringerem Maße Vorteile gezogen als die SP. Meinungsumfragen zufolge hat die SP zeitweise rd. 25 bis $27 \%$ der Wählerintentionen auf sich vereinigen können, während die KP bei rd. $21 \%$ stagnierte. Beide Parteiapparate haben die Sprengwirkung ihrer Profilierungsstrategie offenbar unterschätzt, nicht zuletzt, weil sie deren tief verankerte gesellschaftli. che Bestimmungsgründe nicht berücksichtigt haben. Die KP hat ihre Sperrminorität voll ausgespielt. Sie hat ihre Zustimmung zu einer Vereinbarung, ihre im Linksblock zweitplazierten Kandidaten zurückzuziehen und ihren Wählern zu empfehlen, erstplazierte sozialistische Kandidaten zu wählen, erst nach dem ersten Wahlgang erteilt.

Nach dem ersten Wahlgang, als feststand, daß die Wahlen mit hoher Wahrscheinlichkeit im zweiten Wahlgang nicht mehr gewonnen werden konnten, haben sich die Generalsekretäre und hohen Funktionäre der Parteiapparate am Montag, dem 13. März 1978 von 18.00 bis 23.00 Uhr zusammengesetzt und versucht, thre Profillerungsstrategie durcl: eine Einigungsstrategie zu ersetzen. In rd. 3 Stunden wurde eine überraschend substantielle und ausgewogene politische Erklärung verabschiedet, in der alle Streitpunkte der vergangenen Monate beigelegt, oder ihre Lösung auf später verschoben wurde. Glaubhaftigkeit bei den französischen Wählern konnte mit diesem Dokument nicht wieder erzeugt werden. Wer kann Verständnis dafü aufbringen, daß über politisch-programmatische Probleme, an denen monatelange Ak* tualisierungsverhandlungen gescheitert waren und uber die von September 1977 bis Ende Februar 1978 zwischen den Linksparteien erbittert polemisiert wurde, in fünf Stunden Einigung erzielt werden konnte? Oder anders gefragt: warum wurde diese politische Erklärung, die nach dem ersten Wahlgang in fünf Stunden über die Bühne gebracht werden konnte, nicht bereits im vergangenen Herbst verabschiedet? Die "Dynamik der Einheit" wäre erhalten geblieben und die Wahl mit an Sicherheit grenzender Wahrscheinlichkeit gewonnen worden.

Alle wahlentscheidenden politischen Fehler wurden von den Parteiapparaten ohne Konsultation der Parteibasis auf typisch ,demokratisch-zentralistische "Weise - „national-stalinistisch“, nicht nur in der KP - von den Parteispitzen gemacht. 
Auch nach der selbstverschuldeten Wahlniederlage scheint die Selbstgefälligkeit der Apparate ungebrochen. Mitterrand: „Kurzum, sie (die Kommunisten, G. L.) haben die Niederlage, die sie sich gewünscht haben". Für die KP erklärte Fitermann, Mit. glied des Politbüros, daß alles Übel daher rühre, ,weil sich die sozialistischen Führer in ihren Einschätzungen geirrt haben" (6). Die Apparate der beiden großen Parteien des ehemaligen Linksbündnisses sind vom einwöchigen Einigungszwang zwischen beiden Wahlgängen befreit erneut auf Konfrontationskurs gegangen. Doch regt sich jetzt die Basis in beiden großen Parteien. Die Apparate haben zunehmend Schwierigkeiten, mit den erprobten Techniken die Diskussion zu kanalisieren, reglementieren und schließlich abzuwürgen. Die politischen Folgen der selbstverschuldeten Niederlage sind noch nicht abzusehen. Daß die Parteiapparate erneut den Konfrontatinskurs eingeschlagen haben, kann auf den sich verstärkenden Legitimationsdruck ihrer jeweiligen Parteibasis zurückgeführt werden. Sie versuchen ihre innerparteilichen Schwierigkeiten nach außen gegen den ehemaligen Bündnispartner zu wenden, denn anhaltende Unruhe an der Basis gefährdet die Reproduktionssituation der Ap. parate. - Tiefgreifende gesellschaftliche Veränderungen in Frankreich aber sind ohne Regierungsübernahme durch die wie auch immer vereinigte Linke nicht möglich. Nicht Konfrontations-, sondern nur Einigungsbestrebungen können zu diesem Ziel hinführen. Die „demokratisch-zentralistische" Bestimmung des politischen Kurses, die durchaus nicht ausschließlich Eigenschaft der kommunistischen, sondern in Abstufungen auch der sozialistischen und der sozialdemokratischen Parteien ist zieht im Konfliktfall tendenziell das Reproduktionsinteresse des Apparates gesellschaftlicher Veränderung vor. Gesellschaftliche Veränderung wird deshalb ohne Veränderung der innerparteilichen Entscheidungsstruktur nicht nur in Frankreich nahezu unmöglich.

\section{3) Reformforderungen als Ergebnis der französischen Wirtschaftsentwicklung bis 1973}

De Gaulles Machtergreifung 1958, der Beginn der V. Republik mit ihren zurückgenommenen demokratischen Rechten, hat Frankreich die Möglichkeit einer beschleunigten wirtschaftlichen Entwicklung geöffnet, nachdem die Voraussetzungen für die Beendigung des Algerien-Krieges geschaffen und westafrikanische Kolonialprobleme für Frankreich gelöst worden waren. Schnelle wirtschaftliche Entwicklung drückt sich auch in der vergleichsweise schnellen Verallgemeinerung des Lohnarbeitsverhältnisses in Frankreich aus. Aus der Tabelle 3 wird ersichtlich, daß der Anteil der Lohnabhängigen an den Erwerbstätigen von 1965 bis 1972 immerhin um 5.1 Prozentpunkte angestiegen ist.

Le Monde 15.4 .78 
Tabelle 3: Anteil der Lohnabhängigen an den Erwerbstätigen (in \%)

$\begin{array}{llll} & 1965 & 1968 & 1972 \\ \text { Frankreich } & 74.1 & 75.9 & 79.2 \\ \text { Bundesrepublik } & 80.6 & 81.2 & 84.1 \\ \text { Großbritannien } & 93.3 & 92.9 & 92.2\end{array}$

Quelle: Page, J.-P. (Hrsg.), Profil Economique de la France, Paris 1975, S. 39

Das ist eine -- auch im Vergleich zur Bundesrepublik - schnelle Entwicklung. Nur England, ein seit langem reifes kapitalistisches Industrieland, hat mir rd. $92 \%$ eine erheblich breitere Verallgemeinerung des Lohnarbeitsverhältnisses erreicht.

Die sechziger und die beginnenden siebziger Jahre sind eine Periode, in der die französischen Wachstumsraten des Bruttosozialprodukts leicht über dem EWG-Durchschnitt liegen. In allen Zeitabschnitten der Tabelle 4, einschließlich der beginnenden siebziger Jahre, hat Frankreich höhere Zuwachsraten als die Bundesrepublik. Nur Japan liegt mit Abstand vor den ubrigen relevanten kapitalistischen Industrienationen.

Tabelle 4: Jährliche Wachstumsraten des Bruttosozialprodukts (konstante Preise; in \%)

\begin{tabular}{lcccccc}
\hline & $\begin{array}{c}\text { Durchschn. Durchschn. Durchschn, } \\
1960-72\end{array}$ & $\begin{array}{l}1960-65 \\
1965-72\end{array}$ & & & 1972 & 1973 \\
\hline EWG & 5.1 & 5.3 & 4.9 & 3.5 & 4.1 & 6.0 \\
darunter: & & & & & & \\
$\quad$ Frankreich & 5.8 & 5.9 & 5.7 & 5.5 & 5.5 & 6.3 \\
$\quad$ Italien & 5.1 & 5.3 & 4.9 & 1.4 & 3.1 & 5.9 \\
$\quad$ Bundesrepublik & 4.5 & 5.0 & 4.2 & 2.7 & 3.0 & 5.4 \\
$\quad$ Großbritannien & 2.7 & 3.3 & 2.4 & 1.4 & 3.5 & 5.4 \\
USA & 4.1 & 4.8 & 3.6 & 3.2 & 6.1 & 5.9 \\
Japan & 10.6 & 10.1 & 10.8 & 6.2 & 9.7 & 11.0 \\
\hline
\end{tabular}

Quelle: Page, J.-P. (Hrsg.), S. 33

Verallgemeinerung des Lohnarbeitsverhältnisses, relativ hohe Wachstumsraten des Bruttosozialprodukts charakterisieren die französische Wirtschaftsentwicklung zwischen 1958 und 1972. Wichtig ist es, an dieser Stelle festzuhalten, daß das französische Wirtschaftswachstum mit sprunghaft erhöhten Investitionsquoten nach 1960 verbunden ist, die auf eine Intensivierung der Arbeit hinweisen (7). Entsprechende Verkürzungen der wöchentlichen Arbeitszeit sind jedoch vor 1968, wie ein Vergleich mit den USA und der Bundesrepublik zeigt, ausgeblieben. Die durchschnittliche wöchentliche Arbeitszeit ist mit rd. 44 Stunden im internationalen Vergleich auch 1972 noch immer sehr hoch.

7 Carré, J.-J., Dubois, P., Malinyaud, E., Abrégé de la croissance française, Paris 1973, S. $79 \mathrm{ff}$. 
Tabelle 5: Durchschnittliche wöchentliche Arbeitszeit in der gewerblichen Wirtschaft

\begin{tabular}{llll}
\hline & 1966 & 1969 & 1972 \\
Frankreich & 45.9 & 45.4 & 44.0 \\
Bundesrepublik & 43.7 & 43.8 & 42.7 \\
USA & 41.4 & 40.6 & 40.6 \\
\hline
\end{tabular}

Quelle: Page, J.-P. (Hrsg.), 1975, S. 39

Die Wachstumserfolge, die vor allem durch eine erhebliche Intensivierung der Arbeit erzwungen wurde, sind nur zu einem Teil in Verkürzungen der wöchentlichen Arbeitszeit umgesetzt worden. Die Einkommensverteilung ist ebenfalls relativ unverändert geblieben. Eine neuere Studie weist nach, daß sich die Disparitäten zwischen Empfängern hoher und Empfängern niedriger Löhne von 1954 bis 1972 insgesamt gesehen vergrößert haben. Im Zuge der Mairevolte 1968 hat sich dagegen der Abstand $z$ wischen den hohen Referenzlöhnen und den niedrigen Referenzlöhnen (= Disparität) verringert und ist seitdem wieder geringfügig gestiegen (8). Bei der Einkommensverteilung (Lohneinkommen und sonstige Einkommen) ist Frankreich zusammen mit den USA in der Ländergruppe mit der ungleichmäßigsten Einkommensverteilung, wie eine Studie der OECD aufzeigt, wenn auch hier eine leichte Tendenz zu einer Abnahme der Ungleichheit in den Jahren 1966/67 - 1972 sich durchzusetzen scheint (9). Die Mairevolte hat in Frankreich 1968 von der Studentenrevolte ausgehend auf die Lohnabhängigen übergreifen und zu Massenstreiks und Fabrikbesetzungen fuihren können, weil die Intensivierung der Arbeit in diesem Land bis 1968 keine spürbare Verkürzung der Arbeitszeit und keine Tendenz zu einer als gerechter empfundenen Einkommensverteilung hat sichtbar werden lassen. Die ,Verringerung der Ungleichheit" spielt deshalb auch in allen Parteiprogrammen, nicht zuletzt auch in GRP, eine zentrale Rolle. An diesem Punkt läßt sỉch exemplarisch verdeutlichen, wie Reformforderungen unmittelbar aus den Defizitsituationen der französischen Wirtschaftsentwicklung entstanden sind.

Das gaullistische Konzept der wirtschaftlichen Entwicklung sollte verhindern, daß Frankreich den Anschluß an die heute sogenannte trilaterale Führungsgruppe USA, Japan, Bundestepublik verliert. Eine staatsinterventionistische Industriepolitik wurde eingeleitet, die unter dem Stichwort, industrieller Imperativ " konzipiert wurde (10). Zumindest teilweise Autarkie Frankreichs gehörte zur Zeit de Gaulles noch zum Programm. Frankreich sollte ökonomisch und technologisch wieder auf Weltniveau gebracht werden. Dem Rüstungssektor kam, wenn auch uneingestanden, die Rolle des Antriebsaggregais im forcierten Akkumulationsprozeß zu. Das gaullistische Streben nach nationaler Unabhängigkeit wurde ökonomisch umgesetzt in zu-

\footnotetext{
8 Centre d'Etude des Revenus et des Couts. Dispersion et disparités de salaires en France au cours des vingt dernières années, Paris 1976, S. 147.

9 OECD Economic Outlook, Occasional Studies, Income Distribution in OECD Countries, July 1976 , S. 16 und S. 26.

10 Stoleru, Lionel, Limperatif industriel, Paris 1969, gibt die regierungsoffizielle Version der Ära nach de Gaulle.
} 
sätzliche staatliche Nachfrage nach Rüstungsgitern, die in Frankreich entwickelt und produziert werden sollten. Auf diese Weise entstand die französische Atomstreitmacht, die den Bau von Atomunterseebooten, Kampfflugzeugen, Raketensystemen, elektronischen Steuerungsvorrichtungen etc. nach sich zog und den Auf-bzw. Ausbau entsprechender Industrien einleitete. Es ist jedoch Frankreich nicht gelungen, die entwickelten Technologien in wesentlichem Umfang in den zivilen Bereich zu leiten. Der Aufbau einer nationalen Computerindustrie ist weitgehend gescheitert. Auch im zivilen Flugzeugbau oder in der Atomtechnologie haben sich die französischen Firmen in der internationalen Konkurrenz nicht durchsetzen können. Nur im militärischen Bereich hat Frankreich große „Exporterfolge ${ }^{66}$ erzielt. Frankreich ist heute nach den USA und der UdSSR der drittgrößte Waffenexporteur der Welt (11). Die politische und militärische Instabilität ist auf diese Weise in Krisengebieten, insbesondere in Afrika, vergrößert worden.

Das gaullistische Konzept des industriellen Imperativs hat die außenwirtschaftliche Verflechtung Frankreichs mit den Weltmärkten möglicherweise verzögert, auf Dauer aber sicher nicht einschränken können. Die Exportquote Frankreichs ist im Vergleich zur Bundesrepublik relativ niedrig (siehe Tabelle 6). Sie hat erst ab 1969, d.h. nach de Gaulles Rücktritt und einer relativen Lockerung der Autarkiepolitik stärker zugenommen.

Tabelle 6: Exportquoten kapitalistischer Industrieländer (Anteil der Exporte am Bruttosozialprodukt in \%)

\begin{tabular}{lrrr}
\hline & 1962 & 1969 & 1972 \\
Frankreich & 9.9 & 10.7 & 13.1 \\
Bundesrepublik & 15.4 & 19.6 & 19.4 \\
Italien & 10.5 & 14.0 & 16.7 \\
Japan & 8.3 & 9.4 & 11.8 \\
USA & 3.7 & 3.9 & 4.3 \\
Niederlande & 35.2 & 35.7 & 38.8 \\
\hline
\end{tabular}

Quelle: Nach Page, J.-P. (Hrsg.), S. 285

Bemerkenswert ist die niedrige Exportquote eines großen Landes wie der USA, deren Exportvolumen weltmarktbeherrschend ist. Die Dominanz des Binnenmarktes der USA ist zunächst 1972 noch ungebrochen. 1977 hat sich die Situation der USA infolge hoher Erdölimporte jedoch grundlegend verändert. Am entgegengesetzten Ende der Skala stehen die Niederlande als kleines Land mit extrem hoher Exportquote von rd. $39 \%$ im Jahre 1972, die eine national orientierte Wirtschaftspolitik nahezu ausschließt. Auch Frankreich muß, wie die folgenden Abschnitte zeigen werden, auf Grund seiner zunehmenden außenwirtschaftlichen Verflechtungen mit zunehmendem Verlust an Autonomie bei der Durchsetzung reformorientierter Wirtschaftspolitik rechnen.

Schwächen der französischen Industriestruktur lassen sich an der Exportstruk. tur dieses Landes im Vergleich zur Bundesrepublik kenntlich machen:

11 La France trafiquant d'armes, Paris 1974, S. 5 
Tabelle 7: Exportstruktur (Anteil von Produktgruppen am Gesamtexport in \%)

\begin{tabular}{lcrrr} 
& \multicolumn{2}{c}{ Frankreich } & \multicolumn{2}{c}{ Bundesrepublik } \\
& 1962 & 1971 & 1962 & 1971 \\
\hline Industrieerzeugnisse & 74.0 & 75.9 & 90.2 & 91.1 \\
landwirtschaftliche Erzeugnisse & 14.3 & 17.2 & 2.2 & 3.7 \\
Rohstoffe & 11.7 & 6.9 & 7.6 & 5.2 \\
\hline
\end{tabular}

Quelle: Nach Page, J.P. (Hrsgo), S. 286

Der Anteil der Industrieerzeugnisse an den Gesamtexporten ist mit rd. $75 \%$ im Vergleich zur Bundesrepublik mit rd. $90 \%$ relativ niedrig und bestätigt, daß Frankreich noch immer Schwierigkeiten hat, auf dem Weltmarkt quantitativ und qualitativ konkurrenzfähig zu werden. Das autozentrierte gaullistische Industrialisierungsmodell hat sich auf dem Exportsektor nicht bewährt. Frankreich hat den Anschluß an die trilaterale Führungsgruppe der kapitalistischen Länder bis 1973 nicht gefunden, wenn auch Krisenkonstellationen nach englischem oder italienischem Muster bisher vermieden werden konnten.

Auf dem Hintergrund dieser recht groben und auf Teilbereiche konzentrierten Skizze der französischen Wirtschaftsentwicklung von 1958 bis 1973 läßt sich bereits verdeutlichen, von welchen wirtschaftlichen Struktur- und Entwicklungskomponenten das gemeinsame Regierungsprogramm ausgeht. Es baut auf der Umverteilung von Zuwächsen auf, die aus einem relativ störungsfreien Wachstumsprozeß herrïhren. Die hohen sozialen Kosten dieses Wachstumsprozesses, die von den konservativen Regierungen nicht aufgefangen wurden, sollen durch entsprechende Infrastrukturinvestitionen kompensiert werden. Die zumindest bis 1968 extrem ungleiche Verteilung der Lohneinkommen bzw, der Einkommen im weitesten Sinne sollte durch Erhöhung der Mindestlöhne und Umverteilungsmaßnahmen gemildert werden. Relativ umfangreiche Nationalisierungen sollten die Durchsetzungschancen einer demokratischen Wirtschaftsplanung, insbesondere der Investitionsplanung erhöhen. Extrem autoritäre und letztendlich disfunktionale Entscheidungsstrukturen im Staat, in den nationalisierten und privaten Unternehmen sollten durch Dezentralisierungen und Demokratisierung $u$.ä. abgebaut werden.

Das GRP erweist sich als reformorientierte Wirtschaftspolitik im Verständnis eines linken Keynesianismus (12). Die Einkommensumverteilung bewirkt zweifelsohne eine Verstetigung der effektiven Nachfrage, insbesondere für den in Frankreich umfangreichen Sektor der Klein- und Mittelbetriebe im Konsumgüterbereich. Die Bereitstellung von umfangreichen Infrastrukturinvestitionen im Wohnungsbau, in der medizinischen Versorgung, im Bildungs- und Ausbildungsbereich etc., hätte zumindest teilweise zu Lasten der Rüstungsaufträge erfolgen können und damit ökonomischen Effizienzkriterien besser entsprochen. Unter Einbeziehung des erweiter-

12 Deutschmann, Christoph, Der linke Keynesianismus, Frankfurt 1973, S. 75 ff. gibt eine im wesentlichen auf englische Verhältnisse zugeschnittene Version von Theorie und Politik des linken Keynesianismus, insbes. S. 75 ff. 
ten nationalisierten Sektors wäre ein schrittweiser Übergang zu einer neuen Qualität des Wirtschaftswachstums nicht ausgeschlossen gewesen. Die Anteile der Plansteuerung hätten zu Lasten der Marktsteuerung erhöht werden können. Dank der Verstaatlichung des gesamten Bankensystems wären Finanzierungsprobleme der Programme leichter lösbar gewesen.

Dem französischen Staat kommt bei der Durchführung des GRP eine zentrale Rolle zu. Die theoretische Verankerung im Links-Keynesianismus, soweit bei diesem Programm von theoretischer Fundierung überhaupt gesprochen werden kann, stützt sich auf den Staat als Instrument im Reformprozeß. Die beiden großen Parteien, die SP und die KP, treffen sich in einem nahezu grenzenlosen Optimismus hinsichtlich der Durchsetzungsmöglichkeiten staatlicher Interventionen. Der französische Staat hat zweifelsohne im internationalen Vergleich relativ große Durchsetzungspotentiale. Die Grenzen staatlicher Eingriffe jedoch sind vermutlich viel enger als die Vertragsparteien des GRP es wahrhaben wollten. Die SP zieht ihren Interventionsoptimismus aus technokratischen Konzepten, die ihr von ihren zahlreichen Mitgliedern im öffentlichen Dienst angedient werden. Die KP findet eine Begründung für die Durchsetzbarkeit des Staatsinterventionismus mit Hilfe eines erweiterten nationalisierten Sektors in der Theorie des staatsmonopolitischen Kapitalismus (13). Von konstitutiver Bedeutung für den Interventionsoptimismus sind die Korrespondenz zwischen staatlichen und ,demokratisch-zentralistischen" Entscheidungsstrukturen in den beiden Parteien. Elleinstein sieht diese Beziehung nur in einer Richtung als. Einfluß des französischen staatlichen Zentralismus auf die KP, wenn er sagt: „,Die französische Zentralisation, die Jakobinertradition, erklärt zum Teil einen tiefergehenden und deshalb länger dauernden stalinistischen Einfluß als in der italienischen kommunistischen Partei" (14). - Im Falle einer Regierungsübernahme durch die französischen Linksparteien also wäre die Wahrscheinlichkeit groß gewesen, daß die vertikalen Entscheidungsstrukturen im Staat und in den beiden Parteien sich wechselseitig verfestigen, wenn nicht verstärken. Elleinsteins Versicherung hat dann wenig Gewicht: ,Die KP schlägt den demokratischen Zentralismus nicht als Organisationsmodell für Staat und Gesellschaft vor" (15). Demokratisienung im Staat, verbunden mit der erforderlichen Dezentralisierung, aber ist ohne Demokratisierung innerparteilicher Entscheidungsstrukturen schwer vorstellbar. Es wird an dieser Stelle deutlich, daß der links-keynesianische Interventions-Optimismus in der französischen Parteienkonstellation der Linksunion sich geradezu als ideale theoretische Referenz erweisen muß, denn er bestätigt die bestehende demokratisch-zentralistische Organisation der beiden Linksparteien.

Das GRP scheint, wenn es verwirklicht worden wäre, an die Grenzen des kapitalistischen Systems vorzustoßen. Es bleibt jedoch ein Reformprogramm, das in

13 Boccara u.a., Der staatsmonopolistische Kapitalismus, a.a.O., geben im Kapitel "Nationalisierungen", S. 586 ff., zahlreiche Beispiele eines heillosen Optimismus hinsichtlich der Durchsetzbarkeit staatlicher Interventionen.

14 Elleinstein, Jean, Lettre ouverte en Francais sur la République du Programm Commun, Paris 1977, S. 93

15 ebendort, S. 92 
vielen Bereichen, wie im folgenden Abschnitt zu zeigen sein wird, durchaus nicht ungeeignet ist, die Effizienz der bestehenden kapitalistischen Produktionsweise in Frankreich $z u$ verbessern. Die Frage stellt sich, ob mit einem solchen Frogramm die Grenzen der kapialistischen Ordnung durchbrochen werden können, indem der Reformprozeß über eben diese Grenzen hinausgetrieben wird und ob sozialistische Formen der Produktion, der Verteilung und des menschlichen Zusammenlebens wenigstens in Ansätzen verwirklicht werden können. Einige Elemente zur Beantwortung dieser Frage sollen im nächsten Abschnitt zusammengetragen werden.

\section{4) Ökonomische Grenzen und Möglichkeiten eines reformorientierter Regierungsp rogramms}

Das französische GRP war auf dem Hintergrund eines relativ störungsfreien Wirtschaftswachstums konzipiert. Zur Zeit seiner Unterzeichnung war die Weltwirtschaftskrise, die mit hohen Arbeitslosenquoten erst 1975 voll in Erscheinung tritt, in ihrer vollen Tragweite noch nicht erkannt. Dem GRP wird 1978 deshalb eine weitere Aufgabe zugeschoben, für die es nicht ausgelegt ist. Aus dem Programm müssen Strategien der Krisenüberwindung entwickelt werden. Diese zusätzliche Aufgabe aber drängt das GRP verstärkt in die Argumentationsmuster des linken oder auch des traditionellen Keynesianismus. Es kann nicht davon ausgegangen werden, daß einer linken Regierung die Durchsetzung ihres Reformprogramms durch die Krise erleichtert wird, sondern die Grenzen werden enger gezogen. Die neue Weltwirtschaftskrise tritt in Frankreich stärker noch als in der Bundesrepublik als Stagflation in Erscheinung. Die Arbeitslosigkeit betrifft in Frankreich gegenwärtig nahezu $6 \%$ der Lohnabhängigen und wird 1978 nach einer Prognose der OECD noch um rd. 200000 zunehmen. Trotz harter Deflationspolitik ist es der Regierung Barre nicht gelungen, die Inflationsrate wesentlich unter $10 \%$ zu drücken. Die Wachstums. rate des Bruttosozialprodukts hat sich auf rd. $2.5 \%$ eingespielt. Die französische Leistungsbilanz ist strukturell defizitär. Die Stabilisierungspolitik der Regienung Barre war im Vergleich zur Bundesrepublik nicht erfolgreich. Die Stabilität des sogenannten ,magischen Vierecks ${ }^{66}$ wurde in Frankreich bei weitem nicht erreicht.

Auch in Frankreich ist Geldweristabilität unter der konservativen Regierung inzwischen längst zum Oberziel staatlicher Wirtschaftspolitik avanciert. Monetaristische Konzepte haben dazu Argumentationen geliefert. Hinter den monetaristischen Konzepten kommt die vorkeynesianische Neoklassik wieder zum Vorschein, die Keynes im ersten Buch seiner ,Allgemeinen Theorie" selbst noch einer Kritik unterzogen hatte. Um zu einer neuen Ausgangslage des Akkumulationsprozesses zu gelangen, muß die Kapitalrentabilität wieder hergestellt werden. Die systematische Deflations. politik, die in den entwickelten kapitalistischen Ländern versucht wird, soll zur Verbilligung der Elemente des Kapitals führen. Die Hinnahme einer relativ hohen Arbeitslosigkeit soll dabei die Durchsetzung einer Senkung der Reallöhne bzw. der Lohnquote erleichtern (16). Abgesehen von Militärdiktaturen an der Peripherie der

16 Die Argumentationsmuster der Neoklassik, in denen Flexibilität aller Preise, einschließs- 
Tabelle 8

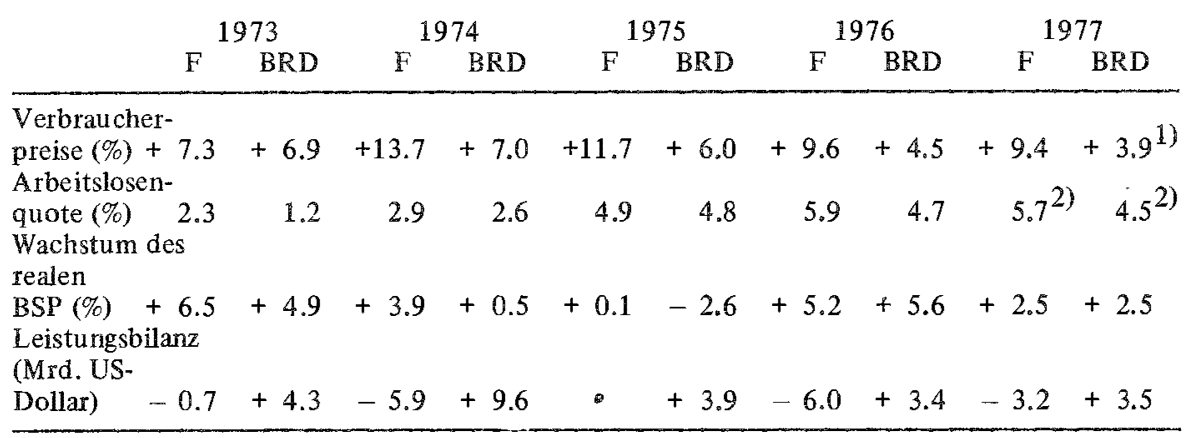

1) 1. Halbjahr 1977

2) 2. Vierteljahr 1977

Quelle: SVR 77/78

kapitalistischen Entwicklung, z.B. Chile oder Argentinien, in denen substantielle Reallohnsenkungen von rd. $25-35 \%$ erzwungen wurden, ist diese Wirtschaftspolitik in entwickelten kapitalistischen Ländern bisher nicht, zumindest aber nicht in ausreichendem Maße, durchsetzbar gewesen. Die Investitionsschwäche, gemessen an der Netto-Anlageninvestition, setzt sich in allen entwickelten kapitalistischen Ländern mit der Ausnahme von Japan fort, d.h. der kapitalistische Akkumulationsprozeß ist nachhaltig gestört.

Grenzen der Durchsetzbarkeit eines reformorientierten Regierungsprogramms lassen sich an zwei Bereichen aufzeigen, im außenwirtschaftlichen und im Bereich der Restriktionen reformorientierter Staatstätigkeit. - Grenzen im außenwirtschaftlichen Bereich können in einem einfachen Szenario deutlich gemacht werden. Eine linke Regierung erhöht die effektive Nachfrage durch zusätzliche Staatsausgaben und substantielle Lohnerhöhungen. Brachliegende Kapazitäten werden erneut ausgelastet und Neuinvestitionen in relevantem Umfang vorgenommen. In Frankreich führte diese Politik zu einer sofortigen sprunghaften Erhöhung der Importe von Rohstoffen und Ausrüstungsgütern. 1977 hatte Frankreich bereits ein Außenhandelsdefizit (Leistungsbilanz) von rd. 3 Mrd. US-Dollar (siehe Tabelle 8). Das Defizit würde schnell ansteigen, die Devisenreserven wären in wenigen Monaten auf einen kritischen Umfang zusammengeschmolzen, der die Aufnahme von Devisenkrediten notwendig machen würde. Damit wird der internationale Währungsfonds eingeschaltet, dessen harte. Auflagenpolitik im oben skizzierten neoklassischen Theorieverständnis verankert ist. Diese Auflagen, die bei der Vergabe von Devisenkrediten vom internationalen Währungsfonds erzwungen werden, greifen - wie etwa das italienische Beispiel zeigt - tief in die nationalstaatliche Wirtschaftspolitik ein. Die betref-

lich der Löhne, auch nach unten, postuliert wird, tangiert in diesem m.E. für die Krisenüberwindung entscheidenden Bereich verschiedene Varianten der marxistischen Akkumulations- bzw. Entwertungstheorien. Siehe hierzu auch Sherman, Howard, J. Stagflation, A Radical Theory of Unemployment and Inflation, New York 1976, S. $89 \mathrm{ff}$. 
fenden Defizite des Staatshaushalts, Tarife öffentlicher Unternehmen, Geldmengenregulierung und vieles andere mehr. Das portugiesische Beispiel zeigt sehr eindringlich die politischen Konsequenzen auf, die aus der Auflagenpolitik des internationalen Währungsfonds entstehen können (17). Sie ist mit reformorientierter Wirtschaftspolitik schlechterdings unvereinbar.

Eine empirische Analyse der Kapitalkreisläufe würde die außenwirtschaftlichen Grenzen nationalstaatlicher Wirtschaftspolitik noch deutlicher nachzeichnen. Hier kann von der Marxschen Analyse ausgehend nur grob und abstrakt skizzierend vorgegangen werden (18). Der Gesamtkreislauf des Kapitals kann zerlegt werden in den Kreislauf des Geldkapitals, des Warenkapitals und des produktiven Kapitals. In entwickelten kapitalistischen Industrieländern sind Geld- und Warenkapitalkreisläufe grundsätzlich grenzüberschreitend und mit außenhandelspolitischen Instrumenten nur begrenzt steuerbar. Die beschleunigte Internationalisierung des Kreislaufs des produktiven Kapitals spätestens seit den sechziger Jahren potenziert die Probleme der Außenwirtschaftspolitik. Transnationale Unternehmen haben tendenziell die Möglichkeit, sich den Zwängen nationalstaatlicher Wirtschaftspolitik zu entziehen, können aber andererseits Steuervergünstigungen und Finanzhilfen vergleichsweise leicht erhalten. Unter diesen Bedingungen werden die Durchsetzungsmöglichkeiten reformorientierter Staatstätigkeit stark eingeschränkt.

Tabelle 9: Umfang des erweiterten öffentlichen Sektors

Anteil in \% an: $\quad$ Vorgesehene Verstaatlichungen Erweiterter öf fentlicher im ,Gemeinsamen Programm" (1) Sektor (2)

\begin{tabular}{|c|c|c|c|c|}
\hline Zahl der Unternehmen & unter & $1 \%$ & unter & $1 \%$ \\
\hline Erwerbsbevölkerung & rund & $4 \%$ & rund & $13 \%$ \\
\hline Bruttoinlandsprodukt & " & $5 \%$ & "، & $14 \%$ \\
\hline $\begin{array}{l}\text { Ausgaben für Forschung } \\
\text { und Entwicklung }\end{array}$ & “ & $13 \%$ & “ & $80 \%$ \\
\hline Industrieinvestitionen & “" & $15 \%$ & “ & $50 \%$ \\
\hline $\begin{array}{l}\text { Beschäftigung in der } \\
\text { Industrie }\end{array}$ & “ & $9 \%$ & “ & $26 \%$ \\
\hline $\begin{array}{l}\text { nicht-landwirtschaftliche } \\
\text { Exporte }\end{array}$ & “ & $20 \%$ & " & $30 \%$ \\
\hline
\end{tabular}

(1) Kommunistische Auslegung

(2) nach den Verstaatlichungen des Gemeinsamen Programms

Quelle: Nach Le Pors, Les Bequilles du Capital, Transfers Etat-Industrie: Critere de Nationalisation, Paris 1977, S. 212

17 Näheres s. Leithäuser, Gerhard, Der Internationale Währungsfonds (IWF) und die eingeschränkte Souveränität nationalstaatlicher Wirtschaftspolitik, Blätter für deutsche und internationale Politik, 3‘78

18 Marx, Karl, Das Kapital, Zweiter Band, MEW 24, Berlin 1972, insbes. Erster Abschnitt. 
Die Frage stellt sich, ob das Nationalisierungsprogramm, so wie es im französischen GRP vorgesehen war, bessere wirtschaftspolitische Steuerungsmöglichkeiten erlaubt hätte. Auf die Darstellung des Nationalisierungsprogramms im einzelnen soll hier verzichtet werden. Anzumerken bleibt, daß der Dissens zwischen SP und KP am 22. September 1977 über den Umfang der Nationalisierungen nach den Annäherungen der Verhandlungspositionenen eine Zahl von Beschäftigten betraf, die erheblich unter 100000 liegen dürfte. Das maximale Gesamtprogramm der Nationalisierungen für Banken, Versicherungen und Filialen der neuen Industriegruppen betrug dabei rd. 650000 Beschäftigte. Der Auslöser des Dissens und des Bruchs in der Linksunion steht in keinem Verhältnis mehr zu den unabsehbaren Folgen der durch ihn verschuldeten Wahlniederlage (19). Tabelle 9 gibt Hinweise über den Umfang des erweiterten öffentlichen Sektors, d.h. einschließlich der im GRP vorgesehenen Nationalisierungen.

Die Tabelle erweckt den Eindruck, als ob über den öffentlichen Sektor in Frankreich, erweitert um die im GRP vorgesehenen Verstaatlichungen, der Akkumulationsprozeß des französischen Kapitals aus der privatwirtschaftlichen Verwertungsrationalität herausgebrochen werden könnte. Die Kontrolle über $80 \%$ der Forschungsund Entwicklungsausgaben in Frankreich, die zu einem wesentlichen, wenn nicht zum überwiegenden Teil bisher bereits aus öffentlichen Mitteln finanziert wurden, scheint eine langfristige Steuerung des qualitativen Wachstumsprozesses zu erlauben. $50 \%$ der Investitionen in der Industrie würde in Frankreich nach den Nationalisierungen im öffentlichen Sektor vorgenommen. Über die Verstaatlichung des Geld- und Kreditsektors, der Versicherungen als Geldkapitalsammelstellen wäre die Investitionsfinanzierung der privat verbliebenen Investitionen, soweit sie über langfristige Kredite getätigt wird, direkt steuerbar. Damit scheint der Schwellenwert zumindest knapp erreicht zu sein, von dem an eine qualitativ andere, demokratisch geplante Entwicklungsstrategie möglich wird. Grenzen aber werden durch die außenwirtschaftliche Verflechtung und durch den Internationalisierungsprozeß des produktiven Kapitals gesetzt, der sich nationalen reformorientierten wirtschaftspolitischen Strategien widersetzt.

Es bleibt fraglich, ob Nationalisierungen eine bessere Steuerung der grenzüberschreitenden Geld- und Warenkapitalströme erlaubt bzw. ob die transnationalen Kreisläufe des produktiven Kapitals bei den komplizierten Beteiligungsverhältnissen besser gesteuert werden können. Denkbar ist auch, daß politisch motivierte Sabotage in Auslandsfilialen zu schweren Störungen in den Produktions- und Zirkulationsprozessen der transnationalen nationalisierten Unternehmen führt. Auch die Demokratisierung von Entscheidungen, so wie sie im GRP vorgesehen war, stößt bei großen transnationalen Unternehmen im In- und Ausland auf große Schwierigkeiten, die sich in jahrelangen blockierenden Gerichtsverfahren niederschlagen können. „Nationalisierungen in einem Land“" werfen bei transnationalen Unternehmen in der ersten Phase schwer lösbare Probleme auf, es sei denn, die nationalisierten Un- 
ternehmen verfolgen eine Geschäftspolitik - wie etwa das nach dem Zweiten Weltkrieg verstaatlichte Automobilunternehmen Renault oder die französischen nationalisierten Banken - die sich von den erwerbswirtschaftlichen Strategien privater Unternehmen nicht unterscheidet.

In Frankreich verfügen die nationalisierten Banken bereits heute über $40 \%$ der Einlagen, die Genossenschaftsbanken über weitere rd. $40 \%$ (20). Die Privatbanken haben mit rd. $20 \%$ der Einlagen dennoch in der Industrialisierungsstrategie seit 1958 die ausschlaggebende Rolle gespielt. Die nationalisierten französischen Banken halten sich in ihrem Geschäftsgebaren streng an die international ublichen privatwirtschaftlichen Standards. Kooperationsabkommen zwischen deutschen Großbanken und französischen Staatsbanken sind dann auch nicht ungewöhnlich. Die französischen nationalisierten Banken sind bisher nicht einmal zur Lösung von Finanzierungsproblemen der französischen Wirtschaftsplanung bevorzugt eingesetzt worden. Der nationalisierte, von der Zentralbank straff geführte Bankensektor hätte die Möglichkeit, über eine selektive Kreditpolitik bei der Verwirklichung der Planziele einer demokratischen Wirtschaftsplanung steuernd einzugreifen und außenwirtschaftlich absichernd zu wirken, indem Kapitalflucht vereitelt wird. - Eines der größten Währungsprobleme jedoch, die Sicherung der Parität des französischen Franken nach der Regierungsibernahme durch die Linke, kann auch ein nationalisiertes Bankensystem nicht garantieren. Einer massiven Spekulation gegen den französischen Franken könnte ein nationalisiertes französisches Bankensystem angesichts des Volumens der Transaktionen nicht länger als einen Tag widerstehen. Kooperation mit ausländischen Zentralbanken und Regierungen ist in einem solchen Fall erforderlich. Sie wird informell organisiert und ist ohne politische und wirtschaftliche Konzessionen nich zu haben. Die Grenzen, die einer reformorientierten Wirtschaftspolitik durch die in den letzten 10 - 15 Jahren stark zunehmende außenwirtschaftliche Verflechtung gesetzt werden, sind hier nur grob angedeutet. Sie spielen in der Diskussion um linke gesellschaftspolitische Strategien noch immer nicht die Rolle, die ihr auf Grund ihrer Bedeutung zukommen würde (21).

Grenzen für eine reformorientierte Wirtschaftspolitik liegen weiterhin in den restriktiven Handlungsspielräumen des Staates. Französische materialistisch orientierte staatstheoretische Untersuchungen, die außerhalb der Theorie des staatsmonopolistischen Kapitalismus stehen, haben versucht, diese Grenzen reformorientierter Staatstätigkeit in den Griff zu bekommen. Auf sie kann in diesem Zusammen-

ebendort, S. 49

21 Auch in der Diskussion in der Bundesrepublik um die Jahresgu tachten des Sachverständigenrates werden die Grenzen, die alternativen wirtschaftspolitischen Strategien durch auBenwirtschaftliche Verflechtung gesetzt werden, nicht hinreichend beruicksichtigt, $z . B$. Baisch u.a., Die Wirtschaftskrise in der BRD, Lewiathan 2/77 oder die Vorschläge zur Beendigung der Massenarbeitslosigkeit, Blätter für deutsche und internationale Politik, 5'77. Auch das diesjährige Gegengutachten dieser Gruppe bringt in diesem Bereich kaum neue Einsichten. Siehe Memorandum '78: Alternativen der Wirtschaftspolitik, Blätter für deutsche und internationale Politik, 5'78. 
hang nicht im einzelnen eingegangen werden (22). Im Gegensatz zur deutschen „Ableitungsd "skussion" treten in der französischen Diskussion politische Probleme stärker in den Vordergrund. In unserem Zusammenhang sollen die Bezüge zur key. nesianischen Theorie besonders hervorgehoben werden.

Mit dem Fortschreiten der neuen Weltwirtschaftskrise traten die keynesianischen Potentiale des GRP stärker in den Vordergrund, die auf eine als zufriedenstellend angesehene Auslastung bestehender Kapazitäten über die Steuenung der effektiven Nachfrage zielt (23). Angesichts der Tatsache, dar in den meisten entwickelten kapitalistischen Industrieländern Defizite der öffentlichen Haushate in den letzien Jahren in größerem Umfang hingenonmen wurden und die Nevverschuldung der Staaten stark angestiegen ist, weist auch das Beispiel Frankreichs und der Bundesrepublik darau hin, daß entgegen monetaristischen Beteuerungen das Gewicht keyne. sianischer Politiken noch immer groß ist. Die Arbeitslosigkeit wäre ohne Haushaltsdefizite zweifellos erheblich höher und die neoklassisch inspirierten Versuche zur Durchsetzung von Reallohnsenkungen nicht zuletzt deshalb weiter vorangetrieben. Die fade Monetarismus-Fiskalismus-Debatte reproduziert in der Theorie die restriktiven Möglichkeiten des Staates, in hochentwickelten kapitalistischen Ländern eine kohärente Wirtschaftspolitik zu konzeptionalisieren und durchzusetzen.

Keynes hat bereits in seinem 1936 verwandten Vorwort zur deutschen Ausgabe der Allgemeinen Theorie zum Interventionsoptimismus beigetragen und gesagt, daßs seine Theorie, ,viel leichter den Verhältnissen eines totalen Staates angepaßt werden "könne und im folgenden Satz einschränkend dargelegt, obwohl seine Theorie für die Verhältnisse angelsächsischer Länder ausgearbeitet sei, bleibe sie dennoch ,auf Zustände anwendbar, in denen die staatliche Führung ausgeprägter ist" (24).

22 Wichtige Arbeiten der jügeren französischen Staatsdiskussion, die hier nicht ausfuhrlich kommentiert werd en können, sind Guilaume, Marc, Le capital et son double, Paris 1975; de Brunkoff, Suzanne, Etat et capital, Paris 1976; Poulantzas, Nicos (Hrsg,), La crise de I'tat, Paris 1976; Association pour la Critique des Sciences Economiques-er Sociales, Sux 1'Etat, Colloque de Nice, 8/9/10. Sept. 1976, Contradictions 1977; Poulantzas, L'etat, le pouvoir, le socialisme, Paris 1978.

23 Einen Hohepunkt der wirtschaftspolitischen Polemik der KPF gegen die Sozialistische Partei stellt der Artikel von Philippe Herzog dar: Vouloir rompre pour changer. Herzog ist einer der führenden Ökonomen der KPF , Mitglied des Zentralkomitees und der Verhandlungsdelegation zur Aktuaiisierung des Gemeinsamen Regierungsprogramms. Dort wird u.a. ausgefüht: ,Weit entfernt, ein Programm, das der Krisen wegen überholt ist, vorzuschlagen, halten wir es für dringend erforderlich, das Gemeinsame Programm zu verwirklichen, gerade weil unser Land eine solche Krise kennt ${ }^{66}$. Uber die Politik der sozialistischen Partei urteil Herzog folgendermaßen: "Es handelt sich darum, das Gemeinsame Programm in der Versenkung verschwinden zu lassen, um eine sozialdemokratische Wirtschaftspolitik zu versuchen, deren Inhalt der Politik von Herrn Barre und Herrn Schmidt gleicht". Le honde, 11.12.1977. Herzog polemisiert in seinem Beitrag gegen einen Artikel von Jacques Gailus, Sekretär der Wirtschaftskommission der Sozialistischen Partei, ,Retour a la raison" (zurick zur Vernunft), Le Monde, 28.11.1977, der seine Lesart nicht ausschliebt. (Ubersetzungen: G. L.)

24 Keynes, John Maynard, Allgemeine Theorie der Beschäftigung, des Zinses und des Geldes, Deutsch von Fritz Waeger, Berlin 1955, S. IX. 
In der Tat setzt die Durchsetzung keynesianischer Wirtschaftspolitik einen starken, totalen Staat voraus. Das nationalsozialistische Deutschland ist denn auch das einzige Land gewesen, das mit wirtschaftspolitischen Strategien, die denen des Keynesianismus verwandt sind, und unter Bedingungen ,ausgeprägter staatlicher Führung“ die Vollbeschäftigung relativ schnell wieder erreicht hat. Umfassende Interventionsstrategien übertragen jedoch die Anarchie privatkapitalistischer Produktion in den Staatsapparat, oder - um mit Kalecki zu sprechen, der wirtschaftliche Krisenzyklus wird in einen politischen Krisenzyklus transformiert (25). Bei Poulantzas wird der Staat zum ,kondensierten Kräfteverhältnis", das klassentheoretisch begriffen ist, und die Interventionspotentiale bestimmt (26). Franz Neumann hat die Grenzen des totalen Staates mit ausgeprägter Führung im Nazi-Deutschland untersucht und die Exzesse des politischen Krisenzyklus ausgelotet (27).

Die sozialistischen ,sozialdemokratischen", aber auch die kommunistischen Staatsvorstellungen (soweit sie in der Orthodoxie der Theorie des staatsmonopolistischen Kapitalismus verankert sind) gehen von schier unbegrenztem Interventions." optimismus aus. Sie sehen im Staat ein Instrument für ihre fortschrittsoptimistischen Machbarkeitsvorstellungen. Sie gründen sich wesentlich auf einen Autonomie-Anspruch des Staates, mit dem er sich „,besondert ", aber nur scheinbar „neben und außer" der zivilen Geselllschaft steht und deshalb zum Instrument der Reformpolitik brauchbar würde. Durch sein Reproduktionsinteresse ist der Staat jedoch auf die Revenuen von Arbeit und Kapital verwiesen und deshalb durch die Möglichkeit von Finanzkrisen in seinem Bewegungsspielraum grundsätzlich begrenzt. Aus dieser Konstellation heraus entwickelt sich ökonomischer Staatsinterventionismus als rationalisierender Mitvollzug der kapitalistischen Entwicklung. Der französische Staat beschränkt seine Interventionen tendenziell auf den Ausgleich von Selbstregulierungsdefiziten bzw. die Verhinderung von Instabilitäten im kapitalistischen Produktions- und Zirkulationsprozeß. Produziert wird damit im Bewußtsein die Vorstellung von Unbeweglichkeit und Beharrungsvermögen des französischen Staates, über dessen Reformbedürftigkeit in allen Parteien, links wie rechts, Einigkeit zu bestehen scheint. Die Wege aber, die zu einer Reform des französischen Staates führen, scheinen ebenso blockiert zu sein wie seine Handlungsfähigkeit. Es wäre gewiß falsch, diese Konstellation zu personalisieren und beispielsweise den leitenden Staatsbeamten und Technokraten anzulasten. Nahezu alle sind auf französischen Elite-Universitäten ausgebildet und verfügen zweifelsfrei über beste Fachkenntnisse und Intentionen, aber auch das, was in Frankreich ,Sens de $1^{\prime}$ Etat ${ }^{6 /}$ (ungefähr: Verantwortlichkeit gegenüber dem Staat) genannt wird (28). Schließlich zeichnet sie ein

25 Kalecki, Michael, Politische Theorie der Vollbeschäftigung, in Meißner, W./Frey, B.S. (Hrsg.), Zwei Aufsätze der Politischen Ökonomie, Marxismus und ökonomische Theorie der Politik, Frankfurt 1974, S. 176 ff.

26 Poulantzas, Nicos, l'Etat, le Pouvoir, le Socialisme, Paris 1978, S. 135 ff.

27 Neumann, Franz, Behemoth, Struktur und Praxis des Nationalsozialismus, 1933-1944, Frankfurt 1977, insbes. S. $615 \mathrm{ff}$.

28 Siehe hierzu die Studie von Birnbaum, Pierre, Les sommets de l'Etat, Essai sur l'elite du pouvoir en France, Paris 1977, insbes. S. $92 \mathrm{ff}$. 
traditionsbewußter Koprsgeist aus, der sie auch dann noch zusammenhält, wenn sie einander politisch bekämpfen (gegebenenfalls auch in den Verhandlungsdelegationen zur Aktualisierung des GRP). Der relative Immobilismus des französischen Staates aber hat eine folgenreiche Konsequenz. Er fördert die Entsendung charismatischer Persönlichkeiten, wie z.B. de Gaulle, an die Spitze des Staates, die dann mit zweifelhaften verfassungsrechtlichen Grundlagen am Staat, insbesondere an den demokratischen Gremien, vorbeiregieren unter Ausnutzung plebiszitärer Verfahren. Auch für den Präsidentschaftskandidaten der Linksunion von 1974, Mitterrand, wurde von der Sozialistischen Partei und sympathisierenden Medien die Konstruktion einer charismatischen Persönlichkeit für unerläßlich erachtet.

Immobilismus charakterisiert die Grenzen des französischen Staatsinterventionismus. Staatsinterventionen, die einander aufheben, subtile Korrekturen und propagandistische Schachzüge dienen dem Management des status quo in der französischen Klassengesellschaft, die auch auf der politischen Ebene in zwei antagonistische Lager geteilt ist. Die Stabilisierungsbemühungen der Regierung Barre, die seit 1976 im Amt ist, sind gescheitert. Das Scheitern ihrer Politik jedoch gesteht sie nicht ein und nimmt für sich die Leerformel in Anspruch, das bestmögliche in der weltweiten Krisensituation für Frankreich getan zu haben, allerdings ohne die selbstgesteckten Ziele erreichen zu können. Auch das Scheitern der Rechten in der Stabilisierungspolitik ist ein wichtiges Indiz für die Restriktionen, denen auch reformorientierter Staatsinterventionismus unterworfen bleibt. Frankreich hat einen umfangreichen totalen Staat im keynesschen Sinn, dessen Zentralismus auch von liberal-konservativer Seite als ,französisches Übel" bezeichnet wird (29). Die wirtschaftspolitischen Interventionspotentiale des französischen Staates scheinen nahe$\mathrm{zu}$ unbegrenzt zu sein: sie reichen von der Bankenüberwachung über Preiskontrollen bis hin zu weitreichenden Polizeivollmachten. Jedoch der französische Staat steht Gewehr bei Fuß. Die Interventionspotentiale können auch nicht annähernd ausgeschöpft werden. Auf diese Weise tritt im französische Staat als ,kondensiertes Kräfteverhältnis" im Sinne von Poulantzas die gesellschaftspolitische Patt-Situation in Erscheinung. Sie ist das Ergebnis der französischen Klassenkämpfe, die die französischen Linksparteien auf der politischen Ebene nicht zu führen wußten.

Grenzen für reformorientierte Wirtschaftspolitik werden vor allem von der zunehmenden außenwirtschaftlichen Verflechtung und hier insbesondere von der beschleunigten Internationalisierung des Kreislaufs des produktiven Kapitals und weiterhin von dẹ Restriktionen, denen staatliche Handlungspotentiale grundsätzlich unterworfen sind, gesetzt. Beide Grenzziehungen aber existieren nicht ohne inneren Zusammenhang nebeneinander, sondern verstärken sich tendenziell gegen-

29 Titel des Buches von Peyrefitte, Alain, Le Mal Francais, Paris 1976. Der Verfasser, parteiloser, aber den Gaullisten nahestehender Justizminister, gibt eine über 500 Seiten reichende Aufzählung der Widersprïche, Ungereimtheiten, Konfusionen etc. des französischen Staates, unter denen französische Staatsmänner von Henri IV bis de Gaulle und Giscard d'Estaing zu leiden hatten. Die Ursachenerklärung und die ,Skizze einer Therapie" aber bleiben mehr als bescheiden. 
seitig. Restriktionen und Ineffizienz außenwirtschaftlicher Staatsinterventionen, z. B. bei der Stabilisierung des Frankenkurses oder der Steuerung transnationaler Unternehmen, selbst wenn sie nationalisiert sind, verstärkt das Gewicht außenwirtschaftlicher Störpotentiale. Die Ausdehnung der Weltmarktabhängigkeit ihrerseits verstärkt die Restriktionen, die reformorientierten Staatsinterventionismus innerhalb der französischen Staatsgrenzen kennzeichnet. Ein Prozeß der Entstaatlichung des ehedem keynesianischen ,totalen Staats" zeichnet sich ab. Staatsfunktionen werden in einem langwierigen, historisch nachweisbaren Prozeß aus der nationalstaatlichen Verfaßtheit herausgebrochen und tendenziell internationalisiert, $\mathrm{d}, \mathrm{h}$. internationalen Institutionen wie dem internationalen Währungsfonds, dem EG-Ministerrat, der UNI, der Weltbank übertragen. In der internationalisierten Sphäre zeichnet sich ein Restaatlichungsprozeß $\mathrm{ab}$, indem die vormals nationalstaatlich organisierten Staatsfunktionen neu gebündelt werden. Völlig offen ist heute, ob dieser Prozeß der nationalen Entstaatlichung bzw. der internationalen Restaatlichung gelingt. Angesichts der Widersprüche und Instabilitäten, die die kapitalistische Produktionsweise kennzeichnen, ist die Neuformierung weltstaatlicher Institutionen zur Steuerung grundsätzlich gestörter Selbstregulierungsmöglichkeiten zwingend erforderlich. Ein wie auch immer geartetes ,Absterben" des bürgerlichen Staates ist in diesem Prozeß nicht erkennbar, wohl aber wird der national verfaßte Staat tendenziell reduziert auf seinen harten Kern - die Repressionsfunktion, d.h. die Ausübung des Anpassungszwangs, mit dem die unmittelbaren Produzenten den streng erwerbswirtschaftlich organisierten Produktions. und Zirkulationsprozessen unterworfen werden.

\section{5) Schlußbetrachtung}

Die selbstverschuldete Wahlniederlage der Linksunion zwingt zu kritischem Überdenken der politischen Fehler. Die Parteiapparate der SP und der KP aber widersetzen sich jeder Form von Selbstkritik. Sie reagieren auf die unruhigere Parteibasis mit einer Verstärkung ihrer Kritik an der jeweiligen Konkurrenzpartei. Damit aber folgen sie ihrem Reproduktionsinteresse und vertiefen die Spaltung der französischen Arbeiterbewegung. Während in der SP die Kritik am Apparat, an Mitterrand und Rocard, den beiden wichtigsten Vertretern des Kurses, der zur Wahiniederlage geführt hat, diffus bleibt, hat Louis Althusser in der bürgerlichen Zeitung „Le Mon$\mathrm{de}^{\text {" }}$ in einer Artikelserie die Linie und die Organisation seiner Partei einer scharfen Kritik unterzogen, weil sie zum Bruch in der Linksunion am 22. September 1977 beigetragen hat (30). Nach der zweifellos pertinenten Kritik fordert der Philosoph die Partei auf, ,aus der Festung herauszukommen " und eine Linie der ,Volksunion" zu verfolgen. In vier Punkten skizziert er eine Alternative, in denen er seine Forderungen zusammenfaßt: 1) Die marxistische Analyse soll ,gerettet ${ }^{66}$ und zu neuem

30 Althusser, Louis, le qui ne peut plus durer dans le parti communiste, Le Monde, 25/26/ 27/28. A pril 1978. 
Leben erweckt werden; 2) die innerparteilichen Entscheidungsstrukturen, d.h. der demokratische Zentralismus, soll reformiert werden; 3) eine konkrete Klassenanalyse der französischen Gesellschaft soll erarbeitet werden; 4) Aufbauend auf diesen drei Forderungen soll die Linie der Volksunion ,ohne Reformismus und Sektarismus $^{66}$ entwickelt werden. - Der Parteiapparat der KP hat die Schlußfolgerungen Althussers zurükgewiesen. Er hat Diskussionen und Kritik in der Partei zwar willkommengeheißen, sich aber geweigert, die Parteipresse für die Diskussion zu öffnen. Der Parteiapparat der KP hofft offenbar auch diesmal, sich den innerparteilichen Veränderungen entziehen zu können. Unterschätzt Althusser die Verfestigung des Apparates? - Bereits 1956 nach dem geheimen Bericht Chruschtschows über die Verbrechen Stalins wäre Gelegenheit gewesen, die Partei zu destalinisieren. Die Parteispitze hat erst Jahre später zugegeben, daß sie im Besitz des Berichts gewesen ist. 1968 hat sich in Frankreich die Möglichkeit eröffnet, die bürgerliche politische und gesellschaftliche Hegemonie in Frankreich zu überwinden. Die Direktion der französischen KP hat diese Gelegenheit nicht wahrgenommen, sondern an der Rekonstruktion des status-quo-ante aktiv mitgearbeitet. Sie ist auch diesmal, wie die letzte Sitzung ihres Zentralkomitees vom 26./27. April 1978 deutlich macht, zur Veränderung ihrer innerparteilichen Entscheidungsstrukturen, die nicht zu Unrecht als „,national-stalinistisch" gelten, nicht bereit. Damit aber hat die eurokommunistische Strategie über die französische Wahlniederlage hinaus in ganz Westeuropa einen schweren Rückschlag erlitten.

Die Diskussion über die Wahiniederlage in Frankreich beschränkt sich bisher auf die politische Ebene und droht dort festgeschrieben zu werden. Die Grenzen der Durchsetzbarkeit eines reformorientierten Programms geraten in den Hintergrund. Sie sind jedoch fur eine Allianz zwischen SP und KP von existentieller Bedeutung. Besonders der nahezu grenzenlose, staatstheoretisch aber keineswegs fundierte Interventionsoptimismus ist politisch explosiv. In ihm treffen sich reformistische und stalinistische Illusionen. Der Interventionsoptimismus entfaltet seine eigene Dialektik, die im Fall einer Regierungsübernahme sich leicht gegen ihre Urheber richten kann. Nachdem sie die Durchsetzbarkeit ihres Programms proklamiert und sich im Wahlkampf als sozialistische oder kommunistische Macher empfohlen haben, könnte ihre reformorientierte Wirtschaftspolitik schnell an objektive Grenzen stoßen und sich ihre Versorechungen leicht als undurchfuhrbar erweisen. In der Wählermeinung stehen die selbsternannten Macher da als Versager, die, einmal an der Regierung, die enttäuschten Massen nur noch mit einer allerdings am längsten funktionsfähigen Staatstätigkeit im Zaum halten können - der Repression. Hier können ihnen ihre Parteierfahrungen aus dem demokratischen Zentralismus nützlich sein, der mit den vertikalen bürokratischen Entscheidungsstrukturen im Staatsapparat eng verwandt ist. Mit der Opposition gegen ihre Wirtschaftspolitik könnten sie versuchen ebenso fertigzuwerden wie mit ihrer innerpartellichen Opposition. Um schließlich angesichts der zunehmenden Internationalisierung des Kapitals und der abnehmenden staatlichen Steuerungspotentiale bei einer Wirtschaftspolitik des rationalisierenden Mitvollzugs zu enden, der der inneren Logik der Kapitalbewegungen entspricht und ihnen zum Durchbruch verhilft. Die vorgeblich reformorientierte Wirtschaftspolitik 
würde sich dann von ihrer konservativen, neoklassisch inspirierten Gegenposition weit weniger als programmatisch vorgesehen unterscheiden.

Die Diskussion über die politischen und wirtschaftspolitischen Strategien, die nach der selbstverschuldeten Wahlniederlage einzuschlagen sind, hat erst begonnen. Es bleibt abzuwarten, ob sie zu einer neuen Qualität reift, die ein solideres Fundament einer Allianz zwischen SP und KP bildet, ohne die gesellschaftliche Veränderungen in Frankreich ausgeschlossen sind.

Analysen zur Entwicklung in Italien in der PROKLA:
Michele Salvati: Der Ursprung der gegenwärtigen Krise in Italien, in: PROKLA
Nr. 4(1972)
Anita Römer: Krise und Gewerkschaftspolitik in Italien,in:PROKLA 19/20/21
(1975)
Altvater/Genth: Eurokommunismus als Strategie in der Wirtschaftskrise in Ita-
lien, in: PROKLA Nr. 26 und 27 (1977)
Kallscheuer/Rafalski/Wenzel: Italien zwischen Stabilisierung und Übergang (I)
in: PROKLA Nr. 29 (1977) 